\title{
On ion-cyclotron-resonance heating of the corona and solar wind
}

\author{
E. Marsch $^{1}$, C. Vocks ${ }^{2}$, and C.-Y. Tu ${ }^{3}$ \\ ${ }^{1}$ Max-Planck-Institut für Aeronomie, Katlenburg-Lindau, Germany \\ ${ }^{2}$ Astrophysikalisches Institut Potsdam, Potsdam, Germany \\ ${ }^{3}$ Department of Geophysics, Peking University, Beijing, China
}

Received: 4 October 2001 - Accepted: 18 January 2002

\begin{abstract}
This paper concisely summarizes and critically reviews recent work by the authors on models of the heating of the solar corona by resonance of ions with high-frequency waves (up to the proton cyclotron frequency). The quasilinear theory of pitch angle diffusion is presented in connection with relevant solar wind proton observations. Hybrid fluid-kinetic model equations, which include wave-particle interactions and collisions, are derived. Numerical solutions are discussed, representative of the inner corona and nearSun solar wind. A semi-kinetic model for reduced velocity distributions is presented, yielding kinetic results for heavy ions in the solar corona. It is concluded that a self-consistent treatment of particle distributions and wave spectra is required, in order to adequately describe coronal physics and to obtain agreement with observations.
\end{abstract}

\section{Introduction}

In the recent past intensive efforts were made to explain features of observed solar EUV (extreme ultraviolet) emission lines of heavy ions. The lines provide, through their widths (broadenings or equivalent ion temperatures) and shifts (ion drift speeds), diagnostic information on the coronal plasma state. Models and concepts were developed to explain these observations as a result of ion heating and acceleration by high-frequency waves in coronal funnels and holes. The theories are based on multi-fluid equations and/or kinetic equations, including the resonance of ions with cyclotron waves. This paper summarizes some of the key model results (obtained in various papers by the authors) and addresses key plasma physics issues related to the problem of wave energy absorption and transport, as well as the evolution of the distribution functions of the particles and spectra of the waves. The basic equations are compiled and numerical results are compared with in situ and remote-sensing observations.

Correspondence to: E. Marsch (marsch@linmpi.mpg.de)
It is shown that a key role is played in the wave-particle kinetics by pitch angle diffusion in the wave frame, in association with plateau formation in the velocity distribution functions. Evidence for this is found in the in situ observations of solar wind protons (from Helios plasma measurements), as well as in recent numerical simulations of heavy ions (such as oxygen or iron). The physics of the wave-particle processes is discussed in light of the existing data and the predictions of quasi-linear theory (QLT). It should be emphasized again at the outset of this article that we will mainly provide here a summarising review of our own recent work.

\section{Evidence for ion-cyclotron-resonance heating}

Spectroscopic determination of the widths of extreme ultraviolet emission lines, as obtained from measurements made on SOHO (Solar and Heliospheric Observatory), indicate that heavy ions in various ionization stages in the corona are very hot (see, e.g. Kohl et al., 1997; Wilhelm et al., 1998; Cranmer et al., 1999), particularly in the polar coronal holes where electrons are relatively cold. The ion kinetic temperatures seem to show some ordering with respect to the local gyro-frequencies (Tu et al., 1998, 1999), indicating that wave-particle processes are important.

It is well-known that the protons in the high-speed solar wind reveal highly skewed and anisotropic velocity distribution functions (VDFs). In particular, the perpendicular proton temperature, which is larger than the parallel proton temperature, has been interpreted as being caused by cyclotron resonant heating (Marsch et al., 1982). Typically, the heavy ions in fast wind move faster and have higher temperatures than the protons, another signature of wave-particle interactions. For reviews of these solar wind phenomena, see, e.g. Marsch (1991) concerning the early in situ measurements made in the ecliptic plane by Helios, and von Steiger et al. (1995), with respect to more recent observations by Ulysses out of the ecliptic. The minor ions can be considered as test 
particles, which probe waves and turbulence and act as tracers of the wave effects.

\section{Pitch angle diffusion of solar wind protons}

\subsection{Quasi-linear diffusion theory}

To explain the detailed kinetics of ion VDFs, the Boltzmann equation, combined with quasi-linear wave theory (see, e.g. Stix, 1992), for wave-particle interactions should be used in models. If the relative wave amplitudes are sufficiently small and the spectra broad in Fourier space, which is the case in the solar wind kinetic regime, QLT is adequate to describe the wave-particle couplings and the evolution of the particle VDFs, as well as of the wave energy spectrum densities (ESDs).

The quasi-linear diffusion equation describes the evolution of the velocity distribution function, $f_{j}\left(v_{\|}, v_{\perp}, t\right)$, of any particle species $j$, for example, in the solar inertial frame of reference, in which the particles and waves are suppose to propagate. Throughout, we will assume that the VDF is normalized to a density of unity. The general diffusion equation for any type of wave, propagating obliquely to the field in a magnetized plasma, has originally been derived by Kennel and Engelmann (1966). It is calculated in a transparent way in the textbook of Stix (1992) and, after Marsch (2002), can be written as

$$
\begin{gathered}
\frac{\partial}{\partial t} f_{j}\left(v_{\|}, v_{\perp}, t\right)=\sum_{M} \sum_{s=-\infty}^{+\infty} \frac{1}{(2 \pi)^{3}} \int_{-\infty}^{+\infty} d^{3} k \hat{\mathcal{B}}_{M}(\mathbf{k}) \\
\times \frac{1}{v_{\perp}} \frac{\partial}{\partial \alpha}\left(v_{\perp} v_{j}\left(\mathbf{k}, s ; v_{\|}, v_{\perp}\right) \frac{\partial}{\partial \alpha} f_{j}\left(v_{\|}, v_{\perp}, t\right)\right)
\end{gathered}
$$

where the pitch angle gradient in the wave frame was introduced. It is given by the velocity derivative

$$
\frac{\partial}{\partial \alpha}=v_{\perp} \frac{\partial}{\partial v_{\|}}-\left(v_{\|}-\frac{\omega_{M}(\mathbf{k})}{k_{\|}}\right) \frac{\partial}{\partial v_{\perp}} .
$$

Note that the well-known, quasi-linear plateau in the VDF implies a vanishing pitch angle gradient, i.e. $\partial / \partial \alpha=0$.

The magnetic field fluctuation spectrum, $B_{M}(\mathbf{k})$, is normalized to the background-field (indicated by $B_{0}$ ) energy density. We define:

$\hat{\mathcal{B}}_{M}(\mathbf{k})=\left(\frac{B_{M}(\mathbf{k})}{B_{0}}\right)^{2}\left(\frac{k_{\|}}{k}\right)^{2} \frac{1}{1-\left|\hat{\mathbf{k}} \cdot \mathbf{e}_{M}(\mathbf{k})\right|^{2}}$.

The symbols used are: $\omega_{M}(\mathbf{k})$ is the frequency of a linear wave mode $M, \mathbf{k}$ is the wave vector, and $\mathbf{e}_{M}(\mathbf{k})$ is the wave polarization vector (Melrose and McPhedran, 1991). The term in the denominator comes from the replacement of the electric field ESD by the magnetic field ESD. It turns out to be physically meaningful to introduce the ion-wave relaxation or collision rate defined as

$$
\begin{aligned}
& v_{j}\left(\mathbf{k}, s ; v_{\|}, v_{\perp}\right)=\pi \Omega_{j}^{2} \delta\left(\omega_{M}(\mathbf{k})-s \Omega_{j}-k_{\|} v_{\|}\right) \\
& \quad \times\left|\frac{1}{2}\left(J_{s-1} e_{M}^{+}+J_{s+1} e_{M}^{-}\right)+\frac{V_{j}(\mathbf{k}, s)}{v_{\perp}} J_{s} e_{M z}\right|^{2} .
\end{aligned}
$$

Here we have also introduced the $s$-order resonance speed and the Bessel functions (with index $s$ ):

$V_{j}(\mathbf{k}, s)=\frac{\omega_{M}(\mathbf{k})-s \Omega_{j}}{k_{\|}}, \quad J_{s}=J_{s}\left(\frac{k_{\perp} v_{\perp}}{\Omega_{j}}\right)$.

The circular components of the wave polarization vector are

$e_{M}^{ \pm}(\mathbf{k})=e_{M x}(\mathbf{k}) \pm i e_{M y}(\mathbf{k})$.

The various symbols used above are: the speed of light is denoted by $c$, the ion charge by $e_{j}$, its charge number by $Z_{j}$, density by $n_{j}$, and mass by $m_{j}$. The plasma frequency of species $j$ is $\omega_{j}^{2}=\left(4 \pi e_{j}^{2} n_{j}\right) / m_{j}$. The ion gyro-frequency, carrying the sign of the charge, reads $\Omega_{j}=\left(e_{j} B_{0}\right) /\left(m_{j} c\right)$. The fractional mass density of species $j$ is defined as $\hat{\rho}_{j}=$ $n_{j} m_{j} / \rho$, with $\rho=\sum_{\ell} n_{\ell} m_{\ell}$. We will make use of the relation $\hat{\rho}_{j} \Omega_{j}^{2}=\omega_{j}^{2} V_{A}^{2} / c^{2}$, where the Alfvén speed is based on the total mass density and defined by $V_{A}^{2}=B_{0}^{2} /(4 \pi \rho)$. Thus, the plasma frequency, $\omega_{j}$, can be expressed through the gyrofrequency and the fractional mass density of each species. Furthermore, we define the parallel, $T_{j \|}$, and perpendicular temperature $T_{j \perp}$, and the corresponding thermal speeds by the relations: $V_{j \|}^{2}=k_{B} T_{j \|} / m_{j}, V_{j \perp}^{2}=k_{B} T_{j \perp} / m_{j}$, where $k_{B}$ denotes Boltzmann's constant.

\subsection{A typical fast solar wind proton distribution}

Helios measurements yielded 3-dimensional proton velocity distributions (Marsch, 1991), which provide a unique opportunity to test the idea of quasi-linear pitch angle diffusion. We present in Fig. 1 one example of a measured VDF, represented as cuts in the $v_{\|}-v_{\perp}$-plane and compare the data with the prediction for plateau formation. Only the innermost contours above the $10 \%$ level of the maximum are shown here. The corresponding plasma parameters are given in Table 1 of the original paper of Marsch and Tu (2001b). The horizontal axis gives $v_{\|}$, and the vertical $v_{\perp}$. The left-hand axis points in the Sun's direction.

The wave dispersion is accounted for in a simplified way, without using the full self-consistent dispersion relation. An empirical instead of theoretical, $V_{p h}=\omega_{M}(\mathbf{k}) / k_{\|}$, phase speed is fitted to the data, thus, obtaining values equal to or somewhat less than the measured $V_{A}$. The black dot marked by number 1 corresponds to the fitted wave phase speed, which was estimated to be $V_{p h}=0.8 V_{A}$. The factor 0.8 is assumed to simulate the dispersion of cyclotron waves and represents the decrease in the phase speed with increasing wave number. We find empirically that $V_{p h}=147 \mathrm{~km} / \mathrm{s}$. The large dashed arc plotted on the left-hand side of the contours is a segment of the circle centered in point 1 . The radius is given by the particle's speed, $w$, in the wave frame: $w=\sqrt{\left(v_{\|}-V_{p h}\right)^{2}+v_{\perp}^{2}}$. Note that a pitch angle in the wave frame is given by the angle formed by the horizontal axis and the line connecting the full black dot with any point on the arc.

We see in Fig. 1 that the outer two arcs almost coincide with the two contours corresponding to fractions of 


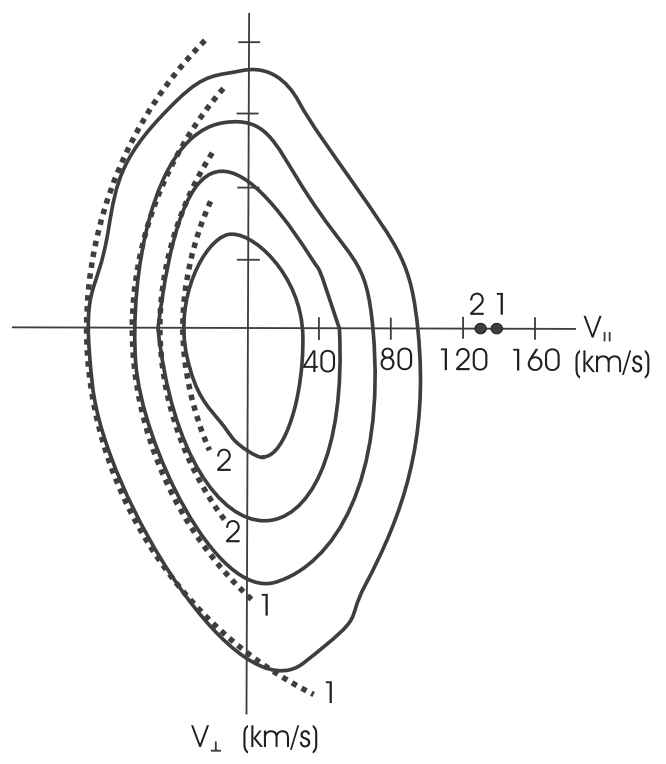

Fig. 1. Comparison of measured proton velocity distributions with the theoretical resonance plateaus, as predicted by QLT. The horizontal axis gives $v_{\|}$and the vertical $v_{\perp}$. The four contours correspond to fractions of $0.8,0.6,0.4,0.2$ of the maximum of the VDF. The thick dotted lines on the left-hand sides are the circular arcs delineating the plateau, whereby the respective centers of the circles are marked on the $v_{\|}$axis by dots (with the same numbers attached to the contours). The dots indicate the locations of $V_{p h}$. The coalignment of the measured contours with the circles of constant particle energy is striking (Marsch and Tu, 2001b).

0.2 and 0.4 of the maximum phase space density, respectively. Over a wide range of about $70^{\circ}$ in pitch angle, the particles, with the tips of their velocity vectors located on the contour, have the same kinetic energy in the wave frame. This is just the cyclotron-resonance plateau as predicted by QLT. To meet the contour at 0.6 of the maximum of the VDF, we need to shift slightly the center of the circle to the point numbered 2, corresponding to a velocity of $0.73 V_{A}=135 \mathrm{~km} / \mathrm{s}$. This arc coincides again with the measured contour, within an interval about $45^{\circ}$ wide in pitch angle in the wave frame. Particles with speeds corresponding to this contour have a smaller $v_{\|}$and thus, are resonant with a higher wave number. The resonant condition can be written as: $\omega\left(k_{\|}\right)-k_{\|} v_{\|}-\Omega_{p}=0$. Since $\omega\left(k_{\|}\right)$is less than the cyclotron frequency, $\Omega_{p}$, and since $k_{\|}$is positive, the outward propagating waves can only resonate with particles having a negative $v_{\|}$. Particles with greater negative $v_{\|}$will be resonant with waves at larger $k_{\|}$or shorter wavelength. For the innermost isodensity contour at 0.8 of the maximum, there is only a $15^{\circ}$ wide range of pitch angles where the measured contour coincides with the arc of constant $w$.

These observations confirm the validity of the basic concepts of QLT, and suggest a further application of QLT to understand the acceleration and heating of ions in the corona and wind. The results also stimulated new ideas and gave further motivation to study the interaction between the ions and Alfvén or oblique ion-cyclotron waves in coronal holes. Generally, plasma waves are dispersive, and there is no preferred reference frame, since the phase speed $V_{p h}$ varies with the wave vector. However, if the waves are weakly dispersive, of a broad band nature and strong enough in intensity, the net result of simultaneous diffusion in closely neighbouring wave frames will be the formation of circle-type contours in velocity space, corresponding to roughly-constant energy surfaces, on which the pitch angle gradient very nearly vanishs. QLT has also recently been applied by Isenberg et al. (2001) and Galinsky and Shevchenko (2000) to model solar wind VDFs by invoking the existence of a plateau.

\section{Wave heating and acceleration rates}

The heating and acceleration rates are obtained by a direct integration over velocity space, as moments of the quasilinear diffusion Eq. (1). By evaluating the delta-function resonances explicitly, the corresponding rates may be cast (after Marsch and Tu, 2001a) in the compact form:

$$
\begin{gathered}
\left(\begin{array}{c}
\frac{\delta}{\delta t} U_{j} \\
\frac{\delta}{\delta t} V_{j \|}^{2} \\
\frac{\delta}{\delta t} V_{j \perp}^{2}
\end{array}\right)=\sum_{M} \frac{1}{(2 \pi)^{3}} \int_{-\infty}^{+\infty} d^{3} k \hat{\mathcal{B}}_{M}(\mathbf{k})\left(\frac{\Omega_{j}}{k_{\|}}\right)^{2} \\
\quad \times \sum_{s=-\infty}^{+\infty} \mathcal{R}_{j}(\mathbf{k}, s)\left(\begin{array}{c}
k_{\|} \\
k_{\|}\left(\begin{array}{c}
\left.V_{j}(\mathbf{k}, s)-U_{j}\right) \\
s \Omega_{j}
\end{array}\right) .
\end{array}\right.
\end{gathered}
$$

Equation (7) expresses the rates in terms of an integral over the normalized magnetic ESD and sums over the mode number, $M$, and resonance-order number, $s$, and the function $\mathcal{R}_{j}(\mathbf{k}, s)$, which also depends on the wave vector $\mathbf{k}$ and, of course, on the VDF, and is obtained from the following definition:

$\mathcal{R}_{j}(\mathbf{k}, s)=\int_{-\infty}^{\infty} d v_{\|} \int_{0}^{\infty} d v_{\perp} v_{\perp}^{2} \frac{k_{\|} v_{j}(\mathbf{k}, s)}{\Omega_{j}^{2}}\left(-\frac{\partial f_{j}}{\partial \alpha}\right)$.

This corresponds to an averaged relaxation rate weighted by the pitch angle gradient at the resonance. As in parallel propagation, $\mathcal{R}_{j}$ for oblique wave propagation can be entirely expressed in terms of the reduced VDFs (see Marsch, 2002), if in the rate $v_{j}$, the dependence on $v_{\perp}$ is smoothed out by replacing this variable by its typical thermal value $V_{j \perp}$. Note that the quantity $\mathcal{R}_{j}$ plays the role of a "wave opacity", using a term from radiative transfer theory. At a given wave vector, the wave absorption vanishes where the pitch angle plateau is formed. Explicit expressions for $\mathcal{R}_{j}$, for example, for a bi-Maxwellian, are given in the paper of Marsch and Tu (2001a). Of course, the rates in Eq. (7) can only be evaluated once the VDF, $f_{j}\left(v_{\|}, v_{\perp}\right)$, of all particle species and the ESD, $\hat{\mathcal{B}}_{M}\left(k_{\|}, k_{\perp}\right)$, of all wave modes involved are known. This complexity is an unavoidable feature of kinetic theory as compared with fluid theory. 


\section{Models for coronal ions}

\subsection{Hybrid fluid-kinetic model equations}

In what follows we consider multi-ion fluid models. Marsch et al. (1982) and Tu and Marsch (2001a) have given detailed descriptions of the multi-fluid equations, which are supplemented by the wave heating and acceleration terms in Eq. (7), describing the wave-particle interactions at the cyclotron resonance. The continuity equation for any species $j$ is:

$n_{j} U_{j} A=C_{j}$,

where $C_{j}$ indicates the conserved particle (number) flux of the ion species $j$. The symbol $A(r)$ is the cross sectional area of the magnetic flux tube. For simplicity, we only quote equations for a one-dimensional model in space where the dependence is on the spatial coordinate $r$, i.e. radial distance from the Sun, or height in the atmosphere. The momentum equation including anisotropic ion pressure can be written:

$$
\begin{array}{r}
\frac{1}{2} \frac{d}{d r} U_{j}^{2}=-\frac{1}{n_{j}} \frac{d}{d r}\left(n_{j} V_{j \|}^{2}\right)-\frac{1}{A} \frac{d A}{d r}\left(V_{j \|}^{2}-V_{j \perp}^{2}\right) \\
-\frac{Z_{j}}{m_{j} n_{e}} \frac{d}{d r}\left(n_{e} k_{B} T_{e}\right)-\frac{V_{\infty}^{2}}{2 r^{2}}+a_{j}^{w}+\frac{\delta}{\delta t} U_{j},
\end{array}
$$

where we have introduced the escape velocity from the Sun: $V_{\infty}=615 \mathrm{~km} / \mathrm{s}$. The acceleration, $a_{j}^{w}$, relates to a wave force associated with low-frequency MHD-type waves, such as Alfvén waves which, as is well-known, exert a force through their wave-pressure gradient. The wave acceleration (see, e.g. McKenzie, 1994) for our one-dimensional model can be written as follows:

$a_{j}^{w}=\frac{d}{d r}\left(\frac{1}{2}\left(\frac{<\delta V>}{V_{A}}\right)^{2}\left[\left(U+V_{A}\right)^{2}-U_{j}^{2}\right]\right)$,

where $U=\sum_{j} \hat{\rho}_{j} U_{j}$ is the plasma's center-of-mass velocity. The electrons enter the momentum equation (without collisions) only through the electric field produced by their pressure gradient. Since quasi-neutrality requires $n_{e}=$ $\sum_{i} Z_{i} n_{i}$, where the sum extends over all ions, one only needs to know the electron temperature profile. In most kinetic ion models, $T_{e}(r)$ is assumed to be given or simply calculated from electron heat conduction. For the actual model profiles, see the subsequent detailed discussions. The internal energy equations, or here, the rate-of-change equations for the ion thermal speeds, then take the form:

$$
\begin{gathered}
\frac{1}{2} \frac{d}{d r} V_{j \|}^{2}=-V_{j \|}^{2}\left(\frac{1}{U_{j}} \frac{d U_{j}}{d r}\right)+\frac{1}{U_{j}} \frac{\delta}{\delta t} V_{j \|}^{2}, \\
\frac{d}{d r} V_{j \perp}^{2}=-V_{j \perp}^{2}\left(\frac{1}{A} \frac{d A}{d r}\right)+\frac{1}{U_{j}} \frac{\delta}{\delta t} V_{j \perp}^{2},
\end{gathered}
$$

where the wave-particle interaction terms appear as sources (heating by wave absorption) or sinks (cooling by wave emission) on the right-hand sides. Without these terms, the double-adiabatic equations result. Equations (9), (10), (12), and (13), sometimes complemented by Coulomb collision terms, or similar equations, have been integrated numerically in various models (Marsch et al., 1982; Isenberg and Hollweg , 1983; Marsch, 1999; Cranmer et al., 1999; Li et al., 1999; Hu et al., 2000; Tu and Marsch, 2001a; Cranmer, 2000, 2001).

\subsection{Hybrid model for solar wind ions}

This section presents some of the numerical results (Marsch, 1999) which have been obtained by integrating the hybrid model equations for spherical geometry of the outer corona and solar wind, following the early work by Marsch et al. (1982). We show numerical solutions, obtained by placing boundary conditions at $20 \mathrm{R}_{\odot}$, for the different moments of $\mathrm{O}^{6+}$ and protons. The VDFs are characterized as drifting bi-Maxwellians in the ion reference frames. Here we did not attempt to integrate through the region of the sonic critical point, but simply started in the near-Sun solar wind. Spectra of broad band LHP and RHP parallel waves were injected at $20 \mathrm{R}_{\odot}$. The abundance $n_{\mathrm{O}} / n_{p}$ was taken as 0.001. The two-ion plasma was initially assumed to be in thermal equilibrium, with $U_{j}=0, T_{j \|}=T_{j \perp}=T_{p \|}$ at $z=20$, where $z=R / \mathrm{R}_{\odot}$ denotes the normalized heliocentric distance. The injected wave spectra is $\mathcal{B}_{\omega}^{ \pm}$, with power laws in frequency for both degrees of polarization, and with $\mathcal{B}_{\omega}^{ \pm}=\mathcal{B}_{\Omega p}^{ \pm}\left(\omega / \Omega_{p}\right)^{-\alpha}$ and a spectral index $\alpha=1.5$. In the models, the spectral index used may vary between $\alpha=1$ and $\alpha=2$, or frequently, the Kolmogorov value, $\alpha=5 / 3$, has been used. For the solar wind observations, see the review by Tu and Marsch (1995) for a comprehensive discussion of the observed spectral slopes.

Figure 2 shows ion thermal speeds and the relative speed of $\mathrm{O}^{6+}$ ions, $\Delta U_{\mathrm{O}}=U_{\mathrm{O}}-U_{p}$, in units of $V_{A}$ (Mach numbers) versus distance from the Sun. The proton relative speed, $\Delta U_{p}$, is not shown here because it is negligibly small due to the very low heavy ion abundance, which means that the protons determine the center-of-mass speed, $U$. Note that the initial phase is characterized by strong cooling of protons parallel and heating of oxygen ions perpendicular to the magnetic field, accompanied by a marked preferential acceleration of $\mathrm{O}^{6+}$. After a few hundred wavelengths, $\lambda_{A}=V_{A} / \Omega_{p}$, the proton-wave interaction saturates at an anisotropy of about $T_{p \perp} / T_{p \|} \simeq 1.4$, in accord with observations in fast wind at $0.3 \mathrm{AU}$ (Marsch, 1991). The oxygen ions also show the typical signature of cyclotron heating with $T_{O \perp} / T_{O \|} \simeq 2$.0. From about $z=21$ onward, the LHP wave particle interaction becomes weaker than it was initially, and the changes become slower.

Inspection of Fig. 2 shows that between $z=23$ and $z=24$, a new situation appears. The $\mathrm{O}^{6+}$ ions now move increasingly into resonance with the, as yet, undamped RHP waves. This resonant interaction leads to a strong acceleration, pronounced perpendicular cooling and parallel heating. Finally, the anisotropy is even reversed with $T_{\mathrm{O} \perp} / T_{\mathrm{O} \|} \simeq 0.9$, and the $\mathrm{O}^{6+}$ ions are trapped at about the Alfvén speed, which represents a limiting value for $\Delta U_{\mathrm{O}}$. In contrast, the 


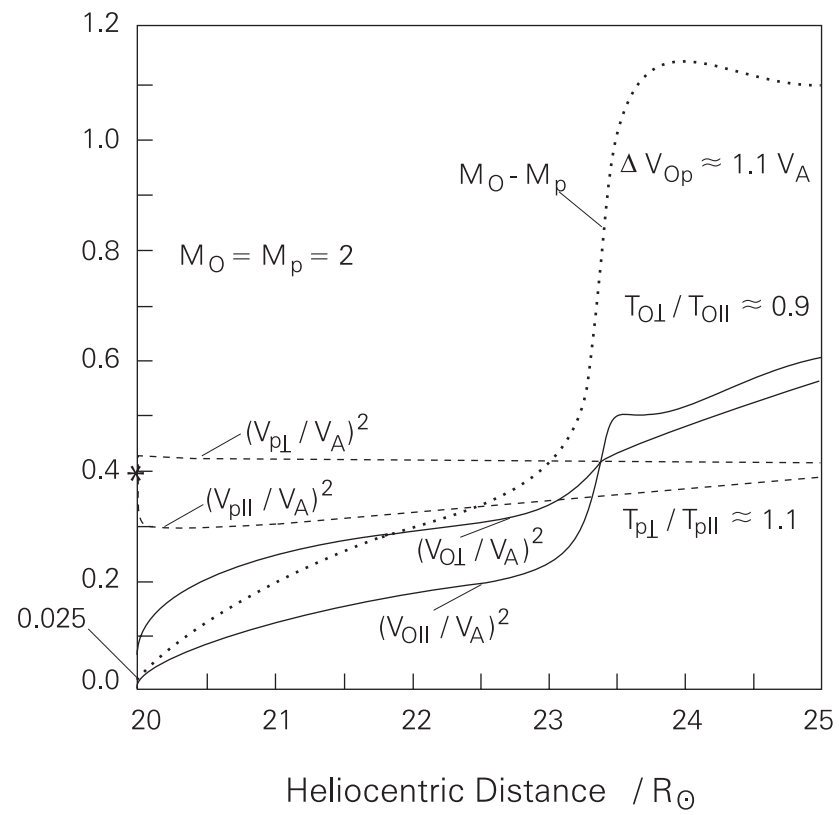

Fig. 2. Hydrogen and oxygen ion thermal speeds squared and oxygen relative speed $\Delta U_{\mathrm{O}}$ normalized to the local Alfvén speed (virtually equal to $M_{\mathrm{O}}-M_{p}$ ) are shown versus heliocentric distance in units of solar radii. The initial values, $\Delta U_{\mathrm{O}}=\Delta U_{p}=0$ and $T_{\mathrm{O}}=T_{p}$, correspond to thermal equilibrium. Note the strong proton cooling parallel to the magnetic field (lower broken curve delineates $\left.\left(V_{p \|} / V_{A}\right)^{2}\right)$. Oxygen ions are accelerated by RHP waves up to about $\Delta U_{\mathrm{O}} \simeq V_{A}$ (dotted line). Their temperatures (continuous lines) finally $(z=24-25)$ show the typical signature of fast-mode wave heating with the anisotropy: $T_{\mathrm{O} \|}>T_{\mathrm{O} \perp}$ (Marsch, 1999).

protons are not significantly affected by the fast waves, but still maintain the signature of the initial perpendicular heating. Qualitatively, these results resemble previous calculations for $\mathrm{He}^{2+}$ (Marsch et al., 1982).

Apparently, $\mathrm{O}^{6+}$ is preferentially heated with respect to the protons. The initial values, $V_{p \|}^{2}=V_{p \perp}^{2}=0.4 V_{A}^{2}$ and $V_{\mathrm{O} \|}^{2}=V_{\mathrm{O} \perp}^{2}=0.025 V_{A}^{2}$, correspond to equal temperatures at $z=20$. After the wave-particle interaction has saturated, the parallel thermal speeds are about equal at $z=23.5$, in good agreement with the observations at $1 \mathrm{AU}$ (von Steiger et al., 1995). Unfortunately, no in situ observations exist concerning the temperature anisotropy of heavy ions.

The inhomogeneity of the expanding wind (note that $V_{A}$ and $\Omega_{p}$ decrease with increasing solar distance) has the effect that the fastest heavy ions in the tails of the distribution move into increasingly stronger resonance with RHP magnetosonic waves. These waves further accelerate the oxygen ions until their differential speed is about $V_{A}$. However, as will be shown in the kinetic model below, there is a problem. The assumed rigidity of the VDF may lead to a gross overestimation of the wave effects, because the resonance function, $\mathcal{R}_{j}(\mathbf{k}, s)$, depends sensitively on the shape of the VDF and should be calculated directly.

In conclusion, some basic qualitative features of the waveparticle interactions are captured by this hybrid model, yet the details may be quantitatively incorrect. What is revealed, however, is that self-consistency is also crucial in describing the radial evolution of the wave ESD, because the wave damping or excitation leads to a reshaping of the original injected wave spectrum or even a complete erosion of the wave power. Such processes must be included in any realistic model.

\subsection{Hybrid fluid-kinetic model for coronal ions}

It has been suggested (Axford and McKenzie, 1997) that magnetic reconnections occurring in the chromospheric network at small-scales may create high-frequency Alfvén waves, and that these waves represent the main energy source for the heating of the solar corona. Following this idea, Tu and Marsch (1997) assumed a wave spectrum in the frequency range from $10^{-4}$ to $200 \mathrm{~Hz}$ at the coronal base. The part of the energy spectrum that is swept by the proton frequency while the wind expands is assumed to be the ion thermal energy source in a two-fluid solar wind model. To produce high-speed wind, the spectrum needs be as high as $106 \mathrm{nT}^{2} \mathrm{~Hz}^{-1}$ at about $200 \mathrm{~Hz}$ (see their model 1). No physical mechanism or observation has yet been presented in support of this assumption. However, the model results are found to be consistent with the proton velocity and the effective temperature observed by UVCS/SOHO (Kohl et al., 1998). Given that these waves exist, they should be absorbed preferentially by the minor heavy ions with low gyrofrequencies, and thus, it is unclear whether there is actually enough wave energy left over in the extended corona for the heating and acceleration of the major solar wind ions, protons and alpha particles, after the multiple absorption by many heavy ions (Cranmer, 2000). Here some selected results of a hybrid kinetic-fluid model (Tu and Marsch, 2001a, $2001 \mathrm{~b}$ ) for the heavy ions are discussed in the context of recent SOHO observations.

The hybrid multi-fluid model includes the self-consistent treatment of the damping of the waves, as well as the associated acceleration and heating of the ions. Tu and Marsch (2001b) showed that if the wave power density is sufficiently large, say about $1000 \mathrm{nT}^{2} \mathrm{~Hz}^{-1}$ at $160 \mathrm{~Hz}$ and $2.5 \mathrm{R}_{\odot}$, then the wave absorption by a prominent minor ion, such as $\mathrm{O}^{+5}$, is small, and most of the wave energy is left for absorption by protons. This is due to the fact that the minor ions are quickly (within several gyro-periods) accelerated and then partially "surf" on the waves. However, if the wave power is too low, say lower than $10 \mathrm{nT}^{2} \mathrm{~Hz}^{-1}$ at $160 \mathrm{~Hz}$ and $2.5 \mathrm{R}_{\odot}$, then damping of the wave power by the $\mathrm{O}^{+5}$ ions is severe, and little wave energy is finally left over for the protons.

Tu and Marsch (2001a) presented several models aimed at explaining the high thermal anisotropy of $\mathrm{O}^{+5}$ ions observed by Kohl et al. (1997). In those models calculations (Tu and Marsch, 2001a), making the assumption of a rigid spectral slope in the dissipation regime, in which case particles can always find waves with suitable resonance frequencies, even an extremely low wave amplitude is sufficient to generate enormous ion heating and acceleration. However, no mech- 

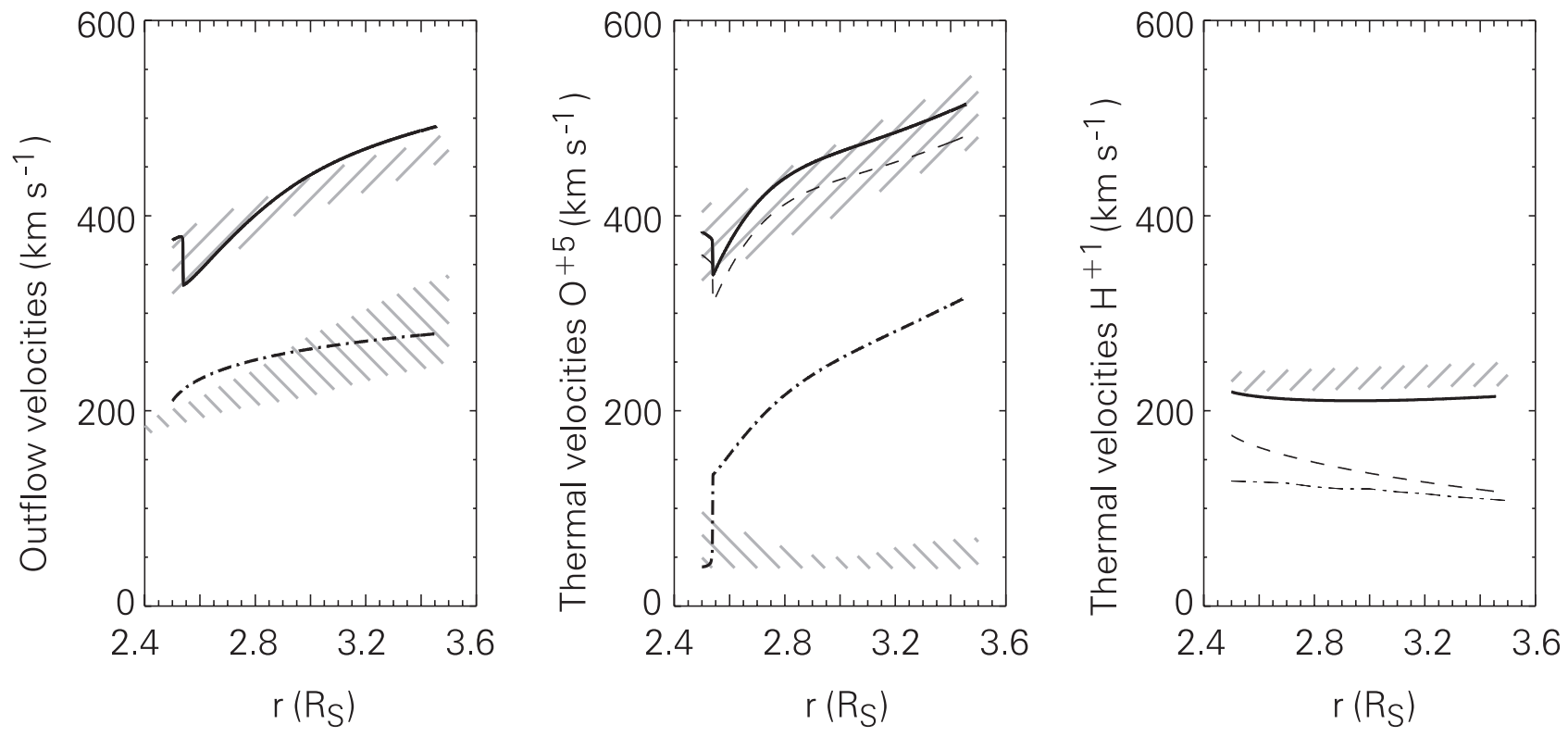

Fig. 3. Comparison of the numerical results UVCS observations. (left) Model results for the radial variations of the outflow velocities of $\mathrm{O}^{+5}$ ions (solid curve) and protons (dash-dotted curve). The observations are indicated by the upper shaded area for $\mathrm{O}^{+5}$ ions and lower for $\mathrm{H}^{0}$ atoms; (middle) The thermal velocities for $\mathrm{O}^{+5}$ ions, including the model results for the effective perpendicular velocity (solid curve), perpendicular thermal speed (dashed curve), and parallel thermal speed (dash-dotted curve). The observed perpendicular effective velocity is shown by the upper shaded area (Cranmer et al., 1999), and the observed parallel thermal speed is shown by the lower shaded area. (right) The model results for the protons: effective perpendicular velocity (solid curve), perpendicular thermal speed (dashed curve), and parallel thermal speed (dash-dotted curve). The observed perpendicular effective thermal velocity for the $\mathrm{H}^{0}$ atoms is indicated by the shaded area. The parallel thermal velocity of $\mathrm{H}^{0}$, assuming that the parallel temperature equals the observed electron temperature, is plotted as a thin dash-dotted curve (Tu and Marsch, 2001a).

anism is known that would guarantee an invariant slope. If the spectrum is allowed to evolve self-consistently, including wave absorption, then a high temperature anisotropy cannot be maintained. The average ion anisotropy at marginal stability in the corona then ranges only between 1 and 2.

Here it is shown how the $\mathrm{O}^{+5}$ ions can become accelerated and heated, while the protons can at the same time absorb a considerable amount of wave energy, if the spectrum is allowed to evolve self-consistently with resonant wave absorption. To achieve this result, a LHP ion-cyclotron wave magnetic ESD of $300 \mathrm{nT}^{2} \mathrm{~Hz}^{-1}$ at $100 \mathrm{~Hz}$ and a solar distance of $2.5 \mathrm{R}_{\odot}$ is required. For simplicity, we take isotropic protons. The oxygen ions are represented by a drifting biMaxwellian. The proton number density, $n_{p}$, at $r=2.5 \mathrm{R}_{\odot}$ is $1.1210^{5} \mathrm{~cm}^{-3}$, which gives an Alfvén velocity at $r=$ $2.5 \mathrm{R}_{\odot}$ of $V_{A}=2000 \mathrm{~km} / \mathrm{s}$. The numerical solution shows that $V_{A}$ decreases to $1500 \mathrm{~km} / \mathrm{s}$ and that $n_{p}$ decreases to $310^{4} \mathrm{~cm}^{-3}$ at $r=3.5 \mathrm{R}_{\odot}$. The proton cyclotron frequency, $f_{p}$, decreases from 460 to $180 \mathrm{~Hz}$. The wave boundary conditions at $r=2.5 \mathrm{R}_{\odot}$ are as follows: The spectral slope is assumed to be $\alpha=2$ (which gives comparatively low power at high frequencies); the ratio between the power density at the oxygen resonance frequency and the background magnetic energy density is then $f_{O^{+5}} P\left(f_{O^{+5}}\right) / B^{2}=210^{-5}$. The low-frequency wave amplitude (in the MHD regime) is $(<\delta B>/ B)^{2}=3.610^{-3}$. To make the model description resemble the real situation in the corona, the effects of the alpha particles on the dispersion relations are included.

The radial evolution of the fluid parameters is shown in Fig. 3. The numerical results are found to be largely consistent with the UVCS observations, except for $V_{O \|}$, which rapidly increases from 50 to $130 \mathrm{~km} / \mathrm{s}$ at $2.6 \mathrm{R}_{\odot}$, and then increases more slowly to $320 \mathrm{~km} / \mathrm{s}$ at $r=3.5 \mathrm{R}_{\odot}$. At $r=2.6$ we also see distinct jumps in the curves of both $V_{O \perp}$ and $V_{O \|}$, indicating that the $\mathrm{O}^{+5}$ ions absorb "negatively" (dashdotted curve) near $r=2.5 \mathrm{R}_{\odot}$, which is to say that they emit wave energy there. These jumps are the result of an instability driven by the huge initial temperature anisotropy, with a ratio of 80 , which was assumed at the lower boundary in compliance with the UVCS observations of oxygen. The anisotropy jumps at $2.6 \mathrm{R}_{\odot}$ from 80 to 4 and then slowly decreases further to the moderate value of 1.2. Such a behaviour of the anisotropy is consistent with earlier calculations made for interplanetary solar wind ions by Marsch et al. (1982) and the results are shown in Fig. 2. According to the UVCS measurements (Kohl et al., 1997), the observed upper limit for $V_{O \|}$ at $3.0 \mathrm{R}_{\odot}$ is $60 \mathrm{~km} / \mathrm{s}$, while $V_{O \perp, \text { eff }}$ is about $300 \mathrm{~km} / \mathrm{s}$. The effective thermal speed is defined as $V_{O \perp \text {,eff }}=\sqrt{2 V_{O \perp}^{2}+(<\delta V>)^{2}}$. As a result, the observed anisotropy is about 25 , which cannot be explained by the hybrid fluid-kinetic model calculations. 
In summary, the hybrid-model results do not permit one to draw convincing conclusions. The observations are not explained satisfactorily. The essential weak point of this model (as indeed, of all the other models with similar ingredients) is the assumption of bi-Maxwellian VDFs for protons and various minor ions. However, the in situ observations (Marsch, 1991) give skewed, two-component protons with large core temperature anisotropy (see Fig. 1), but with a Maxwellian distribution in $v_{\perp}$ (Marsch and Goldstein, 1983). From the EUV observations made by SOHO (Tu et al., 1998), one usually observes line shapes that are primarily Gaussian, and thus, one may infer the coronal heavy ion VDFs to be Maxwellians. In the subsequent section, new attempts to overcome the shortcomings of the hybrid model are described.

\subsection{Semi-kinetic Boltzmann model}

The starting point for a kinetic model is the well-known Boltzmann equation for the distribution function, $f(\mathbf{v}, \mathbf{x}, t)$, which depends on time, $t$, velocity, $\mathbf{v}$, and position vector, $\mathbf{x}$. Including wave-particle interactions and Coulomb collisions, the Boltzmann equation attains the form:

$$
\begin{aligned}
\frac{\partial f}{\partial t} & +\mathbf{v} \cdot \frac{\partial f}{\partial \mathbf{x}}+\left(\mathbf{g}+\frac{q}{m}(\mathbf{E}+\mathbf{v} \times \mathbf{B})\right) \cdot \frac{\partial f}{\partial \mathbf{v}} \\
& =\left(\frac{\delta f}{\delta t}\right)_{w .-p .}+\left(\frac{\delta f}{\delta t}\right)_{\text {Coul } .},
\end{aligned}
$$

where $\mathbf{B}$ is the magnetic, $\mathbf{E}$ is the electric, and $\mathbf{g}$ is the gravitational field. In a multi-component plasma, such a Boltzmann equation has to be used for each particle species considered. Since these equations depend on three spatial and three velocity coordinates, the numerical effort in solving them is considerable and, therefore, simplifications are often made. In the solar corona, all characteristic time scales are small compared to the ion gyro-periods. Thus, it is reasonable to assume gyrotropy. This reduces the number of velocity coordinates from three to two: $\mathbf{v} \longrightarrow\left(v_{\|}, v_{\perp}\right)$. Furthermore, it has been shown by Marsch (1998) and Vocks (2001a) that it is meaningful to reduce the VDFs even further by integration over $v_{\perp}$. This procedure yields (after Dum et al., 1980) two relevant reduced velocity distributions which are defined as follows:

$$
\begin{aligned}
& F_{j \|}\left(v_{\|}\right)=2 \pi \int_{0}^{\infty} d v_{\perp} v_{\perp} f_{j}\left(v_{\perp}, v_{\|}\right), \\
& F_{j \perp}\left(v_{\|}\right)=2 \pi \int_{0}^{\infty} d v_{\perp} v_{\perp} \frac{v_{\perp}^{2}}{2} f_{j}\left(v_{\perp}, v_{\|}\right) .
\end{aligned}
$$

The evolution equations for these reduced VDFs are obtained by taking the corresponding moments of the Boltzmann equation. The wave-particle operator is derived from the basic Eq. (1) and gives a diffusion term:

$$
\begin{gathered}
\frac{\delta}{\delta t} F_{j \|}\left(v_{\|}\right)=\frac{\partial}{\partial v_{\|}} D_{j}\left(v_{\|}\right) \frac{\partial}{\partial v_{\|}} F_{j \perp}\left(v_{\|}\right) \\
-\frac{\partial}{\partial v_{\|}}\left(A_{j}\left(v_{\|}\right) F_{j \|}\left(v_{\|}\right)\right) .
\end{gathered}
$$

The transport coefficients, $D_{j}\left(v_{\|}\right), A_{j}\left(v_{\|}\right)$, and $H_{j}\left(v_{\|}\right)$ are given in the papers of Marsch (1998), Marsch and $\mathrm{Tu}$ (2001a). Their evaluation involves integrations over the wave ESD and the resonant relaxation rate, $v_{j}$, which, for oblique wave propagation, may be approximated by taking it at the typical speed, $v_{\perp}=V_{j \perp}$, as suggested by Marsch (2002). Only then can one meaningfully use the reduced VDFs. We refer to the cited works for the mathematics involved and the algebraic details.

To break the chain of higher-order moments appearing in the original diffusion equation, we make the Gaussian approximation (Marsch, 1998)

$2 \pi \int_{0}^{\infty} d v_{\perp} v_{\perp} \frac{v_{\perp}^{4}}{4} f_{j}\left(v_{\perp}, v_{\|}\right) \approx 2 V_{j \perp}^{2} F_{j \perp}\left(v_{\|}\right)$,

which would be exact for a bi-Maxwellian. Of course, this does not imply that $F_{j \perp}$ is Gaussian itself. Empirical motivation for the factorization of Eq. (18) stems from the solar wind in situ observation, yielding that at any parallel speed the protons' perpendicular speeds are distributed as Gaussian (Marsch and Goldstein, 1983), despite the fact that the VDFs can be skewed, and that there may be proton beams (Marsch, 1991) drifting along the mean field. Thus, the second diffusion equation reads:

$$
\begin{gathered}
\frac{\delta}{\delta t} F_{j \perp}\left(v_{\|}\right)=2 V_{j \perp}^{2} \frac{\partial}{\partial v_{\|}} D_{j}\left(v_{\|}\right) \frac{\partial}{\partial v_{\|}} F_{j \perp}\left(v_{\|}\right) \\
-3 A_{j}\left(v_{\|}\right) \frac{\partial}{\partial v_{\|}} F_{j \perp}\left(v_{\|}\right) \\
-2 F_{j \perp}\left(v_{\|}\right) \frac{\partial}{\partial v_{\|}} A_{j}\left(v_{\|}\right)+H_{j}\left(v_{\|}\right) F_{j \|}\left(v_{\|}\right) .
\end{gathered}
$$

The price to be payed for closure is that the evolution equation for $F_{j \perp}$ is now an integro-differential equation, since the squared perpendicular thermal speed is defined by the first parallel moment of $F_{j \perp}$. In a multi-component plasma, the dependence on $U_{j}$ is also essential, since it cannot be removed by going into the plasma (center-of-mass) frame. Stationary solutions of Eqs. (17) and (19) are obtained if the Gaussian condition,

$F_{j \perp}\left(v_{\|}\right)=V_{j \perp}^{2} F_{j \|}\left(v_{\|}\right)$,

is strictly fulfilled. Using the reduced VDFs, one can construct a gyrotropic, 2-D model VDF by introducing the effective perpendicular thermal speed defined by:

$W_{j \perp}^{2}\left(v_{\|}\right)=\frac{F_{j \perp}\left(v_{\|}\right)}{F_{j \|}\left(v_{\|}\right)}$,

which leads in consistency with the Gaussian approximation to the model VDF

$f_{j}\left(v_{\|}, v_{\perp}\right)=\frac{F_{j \|}\left(v_{\|}\right)}{2 \pi W_{j \perp}^{2}\left(v_{\|}\right)} \exp \left(-\frac{w_{\perp}^{2}}{2 W_{j \perp}^{2}\left(v_{\|}\right)}\right)$.

In the subsequent section, we show an example of this model distribution constructed from the numerical solutions of the 
kinetic equations. Finally, we quote the reduced Boltzmann equations (Vocks and Marsch, 2001a), which attain the form:

$$
\begin{gathered}
\frac{\partial F_{\|}}{\partial t}+v_{\|} \frac{\partial F_{\|}}{\partial r}+\left(\frac{q}{m} E_{\|}-g(r)\right) \frac{\partial F_{\|}}{\partial v_{\|}}+\frac{1}{2 A} \frac{\partial A(r)}{\partial r} \\
\times 2\left(\frac{\partial F_{\perp}}{\partial v_{\|}}+v_{\|} F_{\|}\right)=\frac{\delta F_{\|}}{\delta t}+\left(\frac{\delta F_{\|}}{\delta t}\right)_{\text {Coul. }}, \\
\frac{\partial F_{\perp}}{\partial t}+v_{\|} \frac{\partial F_{\perp}}{\partial r}+\left(\frac{q}{m} E_{\|}-g(r)\right) \frac{\partial F_{\perp}}{\partial v_{\|}}+\frac{1}{2 A} \frac{\partial A(r)}{\partial r} \\
\times 4\left(v_{j \perp}^{2} \frac{\partial F_{\perp}}{\partial v_{\|}}+v_{\|} F_{\perp}\right)=\frac{\delta F_{\perp}}{\delta t}+\left(\frac{\delta F_{\perp}}{\delta t}\right)_{\text {Coul. }} .
\end{gathered}
$$

As before, the symbol $A(r)$ denotes the cross sectional area of the magnetic flux tube. The Coulomb collision terms are not quoted here explicitly. To calculate them for the reduced distribution functions is a tedious task, algebraically as well as numerically. This procedure is described in detail in Vocks (2001a, 2002b). The wave terms have already been given before in Eqs. (17) and (19).

\subsection{Wave spectral transfer}

The diffusion Eqs. (23) and (24) form a closed set. However, to describe the wave-particle interactions self-consistently, it is necessary to calculate the evolution of the wave ESD. This requires a transport or spectral transfer equation, the derivation of which is a subtle task, in particular for a medium such as the supersonic solar wind, in which the Doppler shifts induced by the flow produce important effects. They have been discussed in detail by Tu and Marsch (2001a). A robust theory for wave spectral transfer does not exist for the solar corona, although the problem has been dealt with generally in the book by Melrose and McPhedran (1991), using universal concepts of wave propagation in nonuniform dispersive media.

In particluar, the replenishment of the absorbed wave power by a turbulent cascade in the kinetic regime is not understood. For an accurate description of the energy cascade, the effects of anisotropy of the fluctuation spectrum, as expected in the low-beta corona and of oblique wave propagation in a turbulent subsonic flow, should also be considered. Nonlocal wave interactions in wave number space, such as wave nonlinear decay and modulation, or instabilities associated with kinetic Alfvén waves, may also play a major role in the turbulent energy transfer. For a recent discussion, see the work of Leamon et al. $(1998,2000)$ and Voitenko et al. (2001). These important issues cannot be addressed in this paper. A possible transport equation for the wave spectral energy has been given, for example, by Tu and Marsch (1997) and Vocks and Marsch (2001a). This equation is formulated here for the magnetic $\operatorname{ESD}, \mathcal{B}(\omega)$, in terms of the wave frequency, $\omega$, as a variable instead of the wave vector, $\mathbf{k}$. It can, for instance in the case of Alfvén waves, be written (we omit the wave mode index) as follows:

$\frac{\partial \mathcal{B}}{\partial t}+\frac{1}{A} \frac{\partial}{\partial r}\left(A\left(V_{A}+U\right) \mathcal{B}\right)+\frac{\mathcal{B}}{2 A} \frac{\partial A U}{\partial r}=\frac{\delta \mathcal{B}}{\delta t}$, where $U=\sum_{j} \hat{\rho}_{j} U_{j}$ is the plasma's center-of-mass velocity. The symbol $A(r)$ is the cross sectional area of the magnetic flux tube. The right-hand side represents the wave absorption by the particles, which is obtained by summation of the contributions of all species from Eq. (7), and states total energy conservation, implying that:

$-\int d \omega \frac{\delta \mathcal{B}(\omega)}{\delta t}=\sum_{j} \frac{\rho_{j}}{2} \int d v_{\|}\left[v_{\|}^{2} \frac{\delta F_{j \|}}{\delta t}+2 \frac{\delta F_{j \perp}}{\delta t}\right]$.

The loss due to wave-particle interactions of total wave energy equals the gain of the summed ion kinetic energy. In this equation, no spectral transport of wave energy, for example, by a turbulent cascade, is considered. Such a term can be added on the right-hand side.

\subsection{Semi-kinetic model results for coronal ions}

In a series of papers (Vocks, 2001a, 2002b), (Vocks and Marsch, 2001a, 2002b), semi-kinetic (reduced Boltzmann equation) models have been constructed to describe the subtle kinetic effects of wave-particle interactions in coronal funnels and holes. Some of the relevant equations have been quoted in the previous section. In the simulation results obtained by solving the equations numerically, it is found that heavy ions are heated preferentially and that considerable temperature anisotropies form, the results of which are in accord with SOHO observations. The reduced VDFs of the heavy ions deviate strongly from a Maxwellian, an effect which increases with height due to the decrease in the density, and thus, a decline in the efficiency of Coulomb collisions.

The model plasma discussed here consists of protons, $\mathrm{He}^{2+}$ and $\mathrm{O}^{5+}$, considered as a typical minor ion representating the cumulative effect of all heavy ions. At the lower boundary, the densities, $N_{H e}=0.1 N_{p}$ and $N_{O}=0.001 N_{p}$, are given. Ionization dynamics is not accounted for in the model. The computational domain extends from the transition region over $0.6 \mathrm{R}_{\odot}$ into the lower corona. The results show that in the transition region, the temperatures of protons and $\mathrm{He}^{2+}$ rapidly increase to coronal values, but then stay fairly constant. Their temperatures do not develop anisotropy, but the oxygen ions show a different behaviour. The profile of $T_{O \|}$ is very similar to the temperatures of the lighter ions, but $T_{O \perp}$ is strongly enhanced. It has a local maximum near the lower bound, and it rises continuously to very high values of the order of $10^{7} \mathrm{~K}$ in the corona. Thus, a strong anisotropy with $T_{O \perp}>T_{O \|}$ is formed. This preferred heating of the heavy ions and concurrent formation of a temperature anisotropy is consistent with the SOHO observations (Kohl et al., 1997).

The advantage of the semi-kinetic model is that it enables a more detailed investigation in terms of the underlying VDFs than is possible in fluid or hybrid models (Cranmer, 2000). For this purpose, $F_{\|}\left(v_{\|}\right)$and $F_{\perp}\left(v_{\|}\right)$of the three ion species are plotted at a height of $r=1.44 \mathrm{R}_{\odot}$ in Fig. 4. For protons and $\mathrm{He}^{2+}$ ions, hardly any deviations from a Maxwellian can be seen. For the $\mathrm{O}^{5+}$ ions, $F_{\|}$is also Maxwellian, but $F_{\perp}$ 

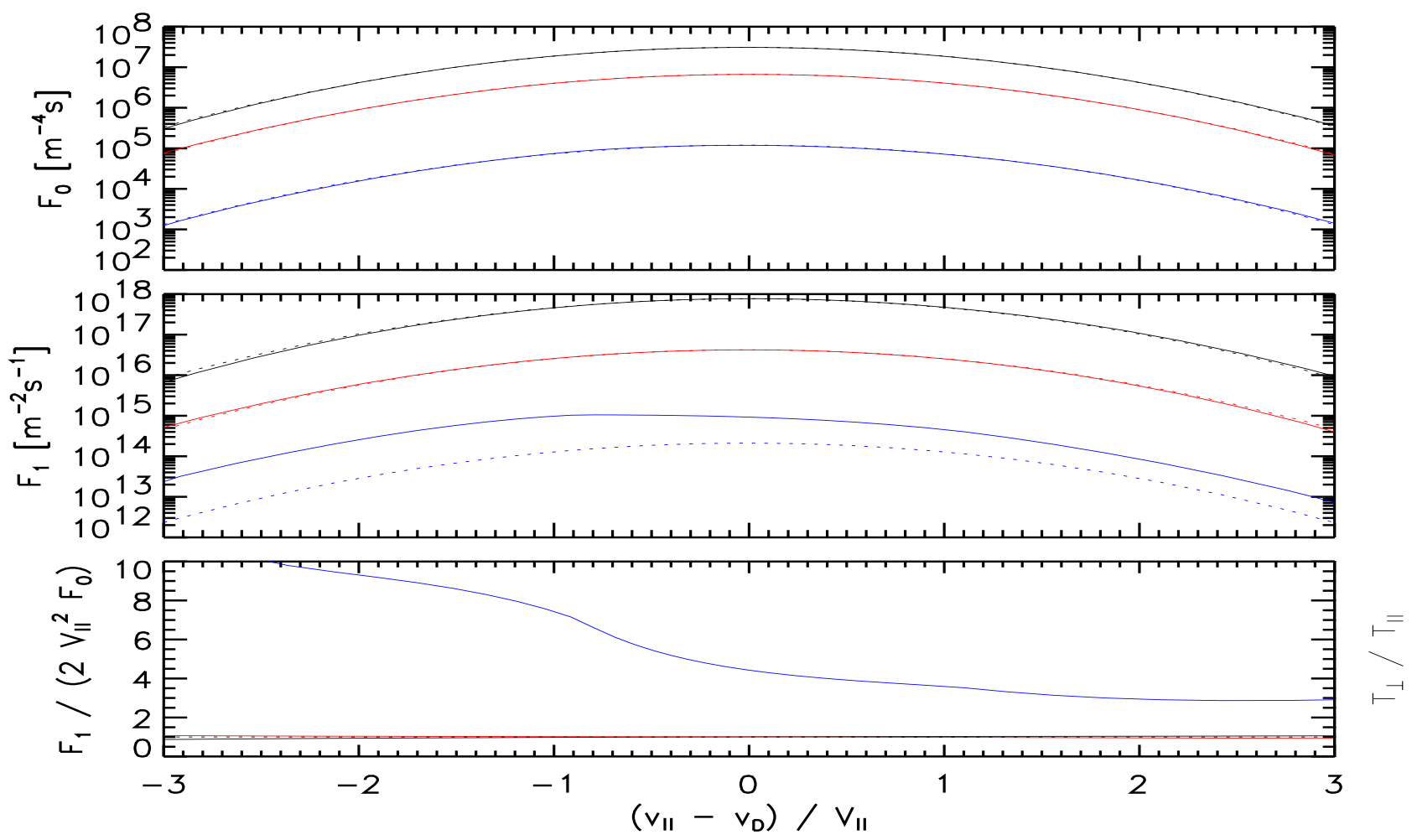

Fig. 4. Reduced VDFs, $F_{\|}$(upper panel) and $F_{\perp}$ (middle panel), of protons (upper lines), $\mathrm{He}^{2+}$ (middle lines) and $\mathrm{O}^{5+}$ (bottom lines) at $1.44 \mathrm{R}_{\odot}$. Also shown are the equivalent Maxwellian VDFs (dotted lines). The lower panel displays the generalized temperature anisotropy of $\mathrm{O}^{5+}$ (Vocks and Marsch, 2001a).

deviates distinctly from a Maxwellian. It is enhanced over $F_{\|}$ for all speeds, with the enhancement being largest at negative speeds in the strong cyclotron-resonance regime.

From the definition of Eq. (21), it follows that the ratio of the two reduced VDFs can be interpreted as a local temperature anisotropy. Therefore, a "generalized temperature anisotropy" function, $A\left(v_{\|}\right)$, may be meaningfully defined as:

$A\left(v_{\|}\right)=\frac{T_{\perp}}{T_{\|}}\left(v_{\|}\right)=\frac{W_{j \perp}^{2}\left(v_{\|}\right)}{V_{j \|}^{2}}$.

For a bi-Maxwellian, $A\left(v_{\|}\right)$is speed independent and identical with the overall constant temperature anisotropy. The anisotropy is plotted in the third panel of Fig. 4. Where the strongest enhancement of $F_{\perp}$ for negative speeds occurs, the anisotropy has its highest values, ranging from about 8 to 10 , and the perpendicular heating and anisotropy become clearly apparent in the reduced VDFs. Apparently, $\mathrm{O}^{5+}$ ions with $v_{\|}<0$ experience the strongest heating. This is a purely kinetic result that cannot be obtained from any fluid model or hybrid model that imposes rigid assumptions on the shape of the VDF.

In the corresponding wave spectrum at $r=1.44 \mathrm{R}_{\odot}$, which is given in Vocks and Marsch (2002b), deep absorption edges occur at and below the ion gyro-frequencies of the species involved. As the cross section area of the magnetic flux tube under consideration increases with height, the magnetic field strength decreases, and so do the gyro-frequencies. For example, the local proton gyro-frequency at $r=1.44 \mathrm{R}_{\odot}$ has a value equivalent to $0.02 \Omega_{p}$ at the lower bound of the computational domain. Therefore, the frequency of a wave that enters the simulation box at the lower bound will, during upward propagation, increase relative to the local ion gyrofrequency. At a certain height, the wave will come into resonance first with the ions having the lowest local resonance frequency. This is the essence of the "frequency sweeping" mechanism discussed by Tu and Marsch (1997). At a certain height, all waves with frequencies above the lowest ion gyro-frequency will have suffered some damping.

Recall that the reduced gyrotropic VDFs, $F_{\|, \perp}$, were introduced to simplify the complexity of the kinetic model. But there is a loss of physical information about the original $f(\mathbf{v})$, namely on its velocity component perpendicular to the field. However, in Eq. (22) we defined a 2-dimensional gyrotropic VDF, which depends on $v_{\|}$in a non-trivial way, reflecting the heating process and the gyro-kinetics of the ions in the magnetic field geometry. Assuming a Maxwellian in $v_{\perp}$, with a spread given by $W_{j \perp}$ of Eq. (21), is consistent with the Gaussian approximation made. A 2-dimensional gyrotropic VDF, $f\left(v_{\|}, v_{\perp}\right)$, can thus be constructed. It is shown in Fig. 5 for $\mathrm{O}^{5+}$ at $r=1.44 \mathrm{R}_{\odot}$. The anisotropy can be recognized by the elliptic deformation of the contours. 


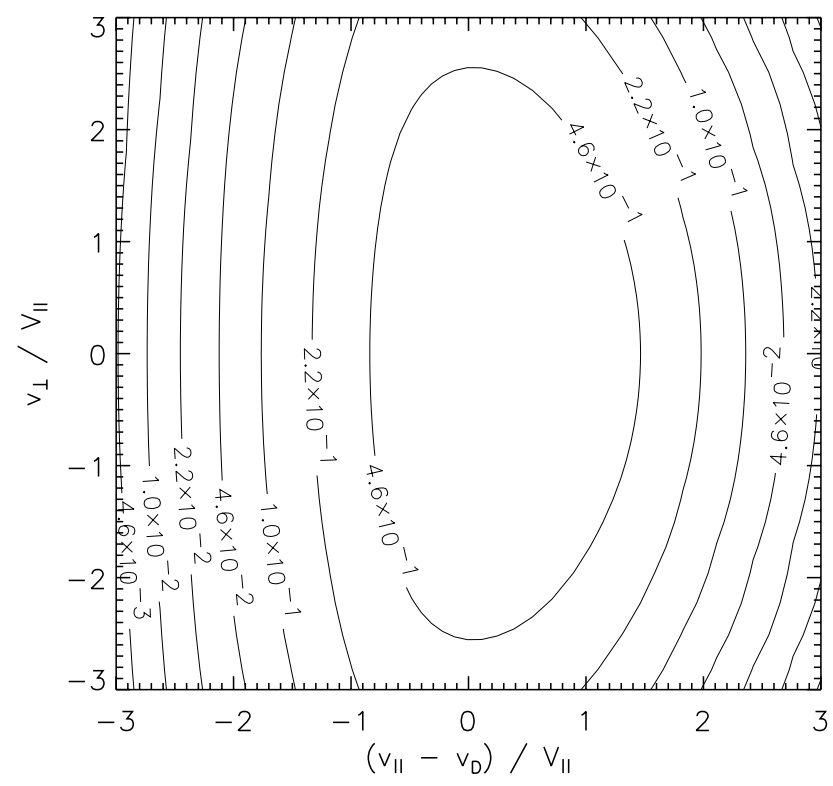

Fig. 5. Two-dimensional gyrotropic model VDF of $\mathrm{O}^{5+}$ ions at $1.44 \mathrm{R}_{\odot}$, displaying a distinct kinetic temperature anisotropy and skewness (heat flux) away from the Sun (Vocks and Marsch, 2002b).

Furthermore, the preferred pitch angle scattering of oxygen ions at negative speeds leads to a skewness of the VDF, which declines in velocity space much faster on the sunward than anti-sunward side. This skewness of the model VDF is a salient feature. A similar skewness has commonly been observed in solar wind proton VDFs (Marsch, 1991). The result again demonstrates the necessity of the kinetic approach to model coronal plasma processes. While the resonant wave heating continues, the contours tend to attain shapes corresponding to the plateaus discussed previously. This result illustrates the main differences with respect to the hybrid models, in which the ions are continuously heated (or cooled by an instability leading to wave emission) when maintaining a rigid bi-Maxwell VDF. In the semi-kinetic model, it was found that the wave heating mechanism prefers the ions with the lowest local resonance frequencies, i.e. $\mathrm{O}^{5+}$ ions with negative $v_{\|}$. However, once a plateau is reached, the growth/damping rate can vanish over a large region of velocity space. This is illustrated in Fig. 6, where $\gamma$ is displayed as a function of the resonance speed of the $\mathrm{O}^{5+}$ ions. The wave growth rate, $\gamma$, can be derived by using the full dispersion relation for waves propagating parallel to the background magnetic field. In Marsch (1998), the dispersion relation for parallel LHP waves is given. The growth rate, for a multi-component plasma, may be written in a form involving only the reduced VDFs:

$$
\frac{\gamma}{\omega}=\left.\frac{\pi}{2} \sum_{j} \hat{\rho}_{j}\left(\frac{\Omega_{j}}{\omega}\right)^{2}\left(-\frac{\Omega_{j}}{k_{\|}} F_{\|}+\frac{\partial F_{\perp}}{\partial v_{\|}}\right)\right|_{v_{\|}=\frac{\omega-\Omega_{j}}{k_{\|}}} .
$$

Apparently, $\gamma$ depends primarily on the number of resonant ions that are able to absorb the waves. Therefore, plotting it normalized to a characteristic frequency, such as $\Omega_{p}$,

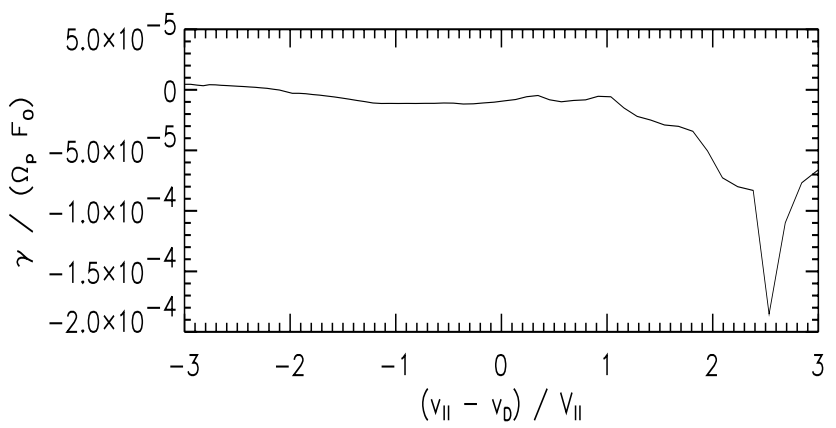

Fig. 6. Normalized growth rate, per resonant particle of $\mathrm{O}^{5+}$, evaluated from model results obtained at $2.46 \mathrm{R}_{\odot}$. The plot displays a distinct flatness of the curve over a wide range of speeds corresponding to a quasi-linear plateau in the VDF (Vocks, 2001a).

does not provide sufficient physical insight into the stability mechanism of the VDF. Especially for large $v_{\|}$, this problem becomes apparent, since at those speeds there are simply no ions that the waves could resonate with. To clarify this issue, $\gamma$ is not only normalized to $\Omega_{p}$, but also to the number of resonant ions given by $F_{\|}\left(v_{\|}\right)$. This function is plotted in Fig. 6 with a startling result. Over a wide range of negative speeds, $\gamma$ is close to zero, meaning that the reduced VDFs have reached marginal stability, at which wave absorption no longer takes place. For positive speeds, $\gamma$ also has some negative values, indicating that here the waves are still being absorbed.

That the ion VDF approaches the limit of stability over a wide range of velocities such that wave absorption ceases, is what one generally expects from resonant pitch angle diffusion leading to plateau formation. That this process takes place in the interplanetary solar wind has been shown in a recent study of the proton VDFs by Marsch and Tu (2001b) discussed above. The strong wave absorption for negative speeds explains the structure of $F_{\perp}$ and the generalized temperature anisotropy $A\left(v_{\|}\right)$, as shown in Fig. 4. For a nearly Maxwellian ion distribution, those ions with $v_{\|}<0$ are heated primarily, as shown in the previous section. With ongoing heating, the oxygen VDF approaches marginal stability, and eventually even those ions with the lowest local resonance frequencies may cease to gain wave energy.

Figure 6 shows that a major fraction of the oxygen ions have a wave absorption coefficient close to zero, i.e. they are transparent for the waves. This is an interesting aspect concerning the wave absorption effects that the numerous different heavy ion species may have in the solar corona, e.g. the 2000 ion species studied by Cranmer (2000). Ions with low cyclotron frequencies could absorb a significant amount of the spectral wave energy in spite of their low densities, but when they reach the limit of stability this absorption ceases, whereupon direct wave heating of ions with higher resonance frequencies is enabled. 


\section{Conclusions}

A short review of the authors' recent work on modelling of the corona is presented with emphasis on key numerical results, remote-sensing and in situ observations and unresolved problems. It has become clear that fluid and hybrid-kinetic models cannot describe the essence of wave-particle interactions. To describe them adequately, in the context of the idea that high-frequency waves heat the solar corona, requires kinetic physics. A semi-kinetic approach with reduced particle VDF and self-consistent wave ESD has provided valuable insights into the coronal plasma physics. Qualitatively new and quantitatively reliable simulation results are obtained which seem to approximate that which is observed. The assumption of fixed shapes of the VDFs or ESDs leads to spurious and unphysical conclusions. The quasi-linear diffusion model captures wave-particle processes in the corona in the case of weak turbulence and also oblique wave propagation. The problem of wave-energy transport and cascading remains unsolved, but certainly deserves great attention in future work.

Acknowledgements. The authors thank the convenors of the Troms $\varnothing$ workshop for their fine organization and for putting together such an interesting programme, which led to stimulating discussions and fruitful scientific exchanges between many colleagues from various countries all over the world.

\section{References}

Axford, W. I. and McKenzie, J. F.: The solar wind, in: Cosmic Winds and The Heliosphere, (Eds) Jokipii, J. R., Sonett, C. P., and Giampapa, M. S., pp. 31-66, Univ. of Ariz. Press, Tucson, 1997.

Cranmer, S. R.: Ion cyclotron wave dissipation in the solar corona: The summed effect of more than 2000 ion species, Astrophys. J., 532, 1197-1208, 2000.

Cranmer, S.: Ion cyclotron diffusion of velocity distributions in the extended solar corona, J. Geophys Res., 106, 24, 937-947, 2001

Cranmer, S. R., Field, G. B., and Kohl, J. L.: Spectroscopic constraints on models of ion cyclotron resonance heating in the polar solar corona and high-speed solar wind, Astrophys. J., 518, 937-947, 1999.

Dum, C. T., Marsch, E., and Pilipp, W. G.: Determination of wave growth from measured distribution functions and transport theory, J. Plasma Phys., 23, 91-113, 1980.

Galinsky, V. L. and Shevchenko, V. I.: Nonlinear cyclotron resonant wave-particle interaction in a nonuniform magnetic field, Phys. Rev. Letters, 85(1), 90-93, 2000.

$\mathrm{Hu}$, Y.-Q., Esser, R., and Habbal, S. R.: A four-fluid turbulencedriven solar wind model for preferential acceleration and heating of heavy ions, J. Geophys. Res., 105, 5093-5111, 2000.

Isenberg, P. A. and Hollweg, J. V.: On the preferential acceleration and heating of solar wind heavy ions, J. Geophys. Res., 88, 3923-3935, 1983.

Isenberg, P. A., Lee, M. A., and Hollweg, J. V.: The kinetic shell model of coronal heating and acceleration by ion-cyclotron waves. 1. Outward-propagating waves, J. Geophys. Res., 106, 5649-5660, 2001.
Kennel, C. F. and Engelmann, F.: Velocity space diffusion from weak plasma turbulence in a magnetic field, Phys. Fluids, 9, 2377f, 1966.

Kohl, J. L., Noci, G., Antonucci, E., et al.: First results from the SOHO UltraViolet Coronagraph Spectrometer, Solar Phys., 175, 613-664, 1997.

Kohl, J. L., Noci, G., Antonucci, E., et al.: UVCS/SOHO empirical determinations of anisotropic velocity distributions in the solar corona, Astrophys. J., 501, L127-L131, 1998.

Leamon, R. J., Matthaeus, W. H., Smith, C. W., and Wong, H. K.: Contribution of cyclotron-resonant damping to kinetic dissipation of interplanetary turbulence, Astrophys. J. Lett., 507, L181-L184, 1998.

Leamon, R. J., Matthaeus, W. H., Smith, C. W., Zank, G. P., and Mullan, D. J.: MHD driven kinetic dissipation in the solar wind and corona, Astrophys. J., 537, 1054-1062, 2000.

Li, X., Habbal, S. R., Hollweg, J. V., and Esser, R.: Heating and cooling of protons by turbulence-driven ion cyclotron waves in the fast solar wind, J. Geophys. Res., 104, 2521-2535, 1999.

Marsch, E.: Kinetic physics of the solar wind, in: Physics of the Inner Heliosphere, (Eds) Schwenn, R. and Marsch, E., Springer Verlag, Heidelberg, Germany, Vol. II, 45-157, 1991.

Marsch, E.: Closure of multifluid and kinetic equations for cyclotron-resonant interactions of solar wind ions with Alfvén waves, Nonlin. Proc. Geophys., 5, 111-120, 1998.

Marsch, E.: Dissipation and wave-ion interaction in the solar wind: Links between fluid and kinetic theory, Nonlin. Proc. Geophys., 6, 149-160, 1999.

Marsch, E.: On resonant interactions of ions with plasma waves in a reduced quasi-linear theory, Nonlinear Processes Geophys., 9, 69-74, 2002

Marsch, E. and Goldstein, H.: The effects of Coulomb collisions on solar wind ion velocity distributions, J. Geophys. Res., 88, 9933-9940, 1983

Marsch, E. and Tu, C.-Y.: Heating and acceleration of coronal ions interacting with plasma waves through cyclotron and Landau resonance, J. Geophys. Res., 106, 227-238, 2001a.

Marsch, E. and Tu, C.-Y.: Evidence for pitch angle diffusion of solar wind protons in resonance with cyclotron waves, J. Geophys. Res., 106, 8357-8361, 2001b.

Marsch, E., Goertz, C. K., and Richter, K.: Wave heating and acceleration of solar wind ions by cyclotron resonance, J. Geophys Res., 87, 5030-5044, 1982.

McKenzie, J. F.: Interaction between Alfvén waves and a multicomponent plasma with differential ion streaming, J. Geophys. Res., 99, 4193-4200, 1994.

Melrose, D. B. and McPhedran, R. C.: Electromagnetic Processes in Dispersive Media, Cambridge Univiversity Press, New York, 1991.

von Steiger, R., Geiss, J., Gloeckler, G., and Galvin, A. B.: Kinetic properties of heavy ions in the solar wind from SWICS/Ulysses, Space Science Rev., 72, 71-76, 1995.

Stix, T. H.: Waves in Plasmas, American Institute of Physics, New York, 1992.

Tu, C.-Y. and Marsch, E.: MHD structures, waves and turbulence in the solar wind: Observations and theories, Space Science. Rev., 73, 1-210, 1995.

Tu, C.-Y. and Marsch, E.: A two-fluid model for heating of the solar corona and acceleration of the solar wind by high-frequency Alfvén waves, Sol. Phys., 171, 363-391, 1997.

Tu, C.-Y. and Marsch, E.: On cyclotron wave heating and acceleration of solar wind ions in the outer corona, J. Geophys. Res., 
106, 8233-8252, 2001a.

Tu, C.-Y. and Marsch, E.: Wave dissipation by ion cyclotron resonance in the solar corona, Astron. Astrophys., 368, 1071-1076, 2001b.

Tu, C.-Y., Marsch, E., Wilhelm, K., and Curdt, W.: Ion temperatures in a solar polar coronal hole observed by SUMER on SOHO, Astrophys. J., 500, 475-482, 1998.

Tu, C.-Y., Marsch, E., and Wilhelm, K.: Ion temperatures as observed in a solar coronal hole, Space Science Rev., 87, 331-334, 1999.

Vocks, C.: Ein kinetisches Modell der Ionen in koronalen Löchern mit Welle-Teilchen-Wechselwirkung und Coulomb-Stößen, PhD Thesis, Technische Universität Braunschweig, Göttinger Beiträge zur Physik, Band 8, Duerkohp \& Radicke, Wissenschaftliche Publikationen, Germany, 2001a.

Vocks, C.: A kinetic model for ions in the solar corona including wave-particle interactions and Coulomb collisions, Astrophys. J., 568, 1017-1029, 2002b.

Vocks, C. and Marsch, E.: A semi-kinetic model of wave-ion interaction in the solar corona, Geophys. Res. Lett., 28, 1917-1920, 2001a.

Vocks, C. and Marsch, E.: Kinetic results for ions in the solar corona with wave-particle interactions and Coulomb collisions, Astrophys. J., 568, 1030-1042, 2002b.

Voitenko, Yu., Goossens, M., and Marsch, E.: On the role of ioncyclotron kinetic Alfvén waves in the solar wind: Results from Helios and expectations for Solar Orbiter, ESA-SP 493, 411416, 2001.

Wilhelm, K., Marsch, E., Dwivedi, B. N., et al.: The solar corona above polar coronal holes as seen by SUMER on SOHO, Astrophys. J., 500, 1023-1038, 1998. 